\title{
Distinction of pulmonary large cell neuroendocrine carcinoma from small cell lung carcinoma: a morphological, immunohistochemical, and molecular analysis
}

\author{
Kenzo Hiroshima ${ }^{1,2}$, Akira Iyoda ${ }^{3}$, Takashi Shida ${ }^{4}$, Kiyoshi Shibuya ${ }^{3}$, Toshihiko Iizasa ${ }^{3}$, \\ Hirohisa Kishi ${ }^{5}$, Tohru Tanizawa ${ }^{2}$, Takehiko Fujisawa ${ }^{3}$ and Yukio Nakatani ${ }^{1,2}$ \\ ${ }^{1}$ Department of Diagnostic Pathology, Graduate School of Medicine, Chiba University, Chiba, Japan; \\ ${ }^{2}$ Department of Pathology, Chiba University Hospital, Chiba, Japan; ${ }^{3}$ Department of Thoracic Surgery, \\ Graduate School of Medicine, Chiba University, Chiba, Japan; ${ }^{4}$ Department of Molecular Pathology, \\ Graduate School of Medicine, Chiba University, Chiba, Japan and ${ }^{5}$ Department of Pathology, \\ Narita Red Cross Hospital, Chiba, Japan
}

\begin{abstract}
The distinction between pulmonary large cell neuroendocrine carcinoma and small cell carcinoma is difficult in some cases. Some propose that these carcinomas should be classified as one high-grade neuroendocrine carcinoma. We examined biological features of small cell carcinoma $(n=23)$, large cell neuroendocrine carcinoma $(n=17)$, and classic large cell carcinoma $(n=12)$. The average ratio of nuclear diameter of the tumor cells to that of lymphocytes for small cell carcinoma was smaller than that for large cell neuroendocrine carcinoma $(P<0.0001)$. The frequencies of the expressions of CD56, mASH1, TTF-1, and p16 were higher and that of NeuroD was lower in small cell carcinoma than in large cell neuroendocrine carcinoma. The frequency of loss of heterozygosity at $3 p$ was higher in high-grade neuroendocrine carcinomas than in classic large cell carcinoma $(P=0.0002)$. Allelic losses at D5S422 $(5 q 33)$ were more frequent in small cell carcinoma than in large cell neuroendocrine carcinoma $(P=0.0091)$. Mean fractional regional loss indices of the tumors were $0.38,0.65$, and $\mathbf{0 . 7 2}$ for patients with classic large cell carcinoma, large cell neuroendocrine carcinoma, and small cell carcinoma, respectively $(P=0.0003)$. Five-year overall survivals of patients with classic large cell carcinoma, large cell neuroendocrine carcinoma and small cell carcinoma in stage I were $67,73,60 \%$, respectively. Patients with NeuroD expression had better survivals, and those with p63 expression had poorer survivals in large cell neuroendocrine carcinoma. Patients with TTF-1 expression had poorer survivals in small cell carcinoma. Our data suggest that large cell neuroendocrine carcinoma and small cell carcinoma are different morphologically, phenotypically, and genetically, although there are some overlapping features. Although further studies are needed to analyze the biological behavior of high-grade neuroendocrine carcinomas including sensitivity to chemotherapy, the pathological distinction of large cell neuroendocrine carcinoma from small cell carcinoma may be necessary to treat the patients with neuroendocrine tumors.
\end{abstract}

Modern Pathology (2006) 19, 1358-1368. doi:10.1038/modpathol.3800659; published online 7 July 2006

Keywords: large cell neuroendocrine carcinoma; small cell lung carcinoma; mASH1; NeuroD; TTF-1; $p 16$

Tumors of the lung with neuroendocrine morphology by light microscopy comprise a spectrum of tumor types with different biology and clinical features. The morphologic types include low-grade typical carcinoid, intermediate-grade atypical carci-

Correspondence: Dr K Hiroshima, MD, Department of Diagnostic Pathology, Graduate School of Medicine, Chiba University, 1-8-1 Inohana, Chuo-ku, Chiba 260-8670, Japan.

E-mail: kenzo@faculty.chiba-u.jp

Received 29 August 2005; revised 12 June 2006; accepted 13 June 2006; published online 7 July 2006 noid, high-grade large cell neuroendocrine carcinoma, and small cell carcinoma.

The first WHO classification of small cell carcinoma in 1967 included four subtypes: lymphocytelike, fusiform, polygonal, and others. ${ }^{1}$ The second WHO classification in 1981 changed 'lymphocytelike' type to 'oat cell carcinoma'; 'fusiform cell' type and 'polygonal cell' type were combined and changed to 'intermediate cell' type; and 'others' was changed to 'combined oat cell carcinoma'. ${ }^{2}$ The third WHO classification in 1999 dropped the terms 'oat cell carcinoma' and 'intermediate cell' type, and 
all tumors with pure histology were called small cell carcinomas. $^{3}$

Tumor cells of small cell carcinoma are round, oval, or spindle-shaped; usually less than the size of three small resting lymphocytes, and have scant cytoplasm, finely granular chromatin, and absent or inconspicuous nucleoli. ${ }^{3}$ The tumor cells of large cell neuroendocrine carcinoma are generally large and polygonal with moderate to abundant cytoplasm; nuclear chromatin is coarsely granular and nucleoli are prominent. ${ }^{3,4}$

Distinguishing large cell neuroendocrine carcinoma from small cell carcinoma can be difficult in some cases, however. In a study by Travis et $a l^{5}$ there was unanimous diagnostic agreement on $70 \%$ of small cell carcinomas and $40 \%$ of large cell neuroendocrine carcinomas in surgically resected neuroendocrine tumors of the lung that were reviewed independently by five pulmonary pathologists. Most of the disagreements concerned the distinction between small cell carcinoma and large cell neuroendocrine carcinoma. Marchevsky et $a l^{6}$ reported that small cell carcinoma and large cell neuroendocrine carcinoma had a continuum of cell size. They and others suggested that the tumors should be combined into a single group in daily practice as a high-grade neuroendocrine carcinoma. ${ }^{6-8}$

Small cell carcinomas respond initially to chemotherapy, but they frequently relapse and metastasize; whereas surgery is advocated for the treatment of patients with large cell neuroendocrine carcinoma, but survival rates are poor. However, it has been reported that patients with stage I large cell neuroendocrine carcinoma, who were treated with either neoadjuvant or postoperative adjuvant chemotherapy, had significantly higher survival rates than patients treated only with surgery. ${ }^{9}$

The aim of this study was to elucidate the morphological, phenotypical, and genetic characteristics of large cell neuroendocrine carcinoma and compare them with those of small cell carcinoma, to determine the biological differences of these two tumors, and to evaluate the utility of these markers for the diagnosis of neureondocrine tumors and the prognosis according to the expression of these proteins.

\section{Materials and methods}

\section{Tumor Specimens}

We reviewed the histologic features of large cell neuroendocrine carcinoma reported in our recent paper $^{10}$ and the cases diagnosed after the publication of the manuscript. Tumors were classified according to the WHO classification system for lung carcinoma. ${ }^{3,11}$ We analyzed 17 cases of large cell neuroendocrine carcinoma in stage I (T1NOM0 or T2N0M0): a tumor that does not invade the adjacent organs (T1 or T2), has no regional lymph node metastasis (No), and no distant metastasis (M0). One case of large cell neuroendocrine carcinoma was combined with squamous cell carcinoma. We also analyzed 12 cases of classic large cell carcinoma in stage I, nine cases of carcinoid tumors (seven typical carcinoids and two atypical carcinoids) in stage I, and 23 cases of small cell carcinoma with limited disease (17 cases in stage I and six cases in stages II-IV). Three small cell carcinomas were combined with squamous cell carcinoma, and one was combined with large cell neuroendocrine carcinoma. All tumors, except one large cell neuroendocrine carcinoma, were resected surgically at Chiba University Hospital between 1987 and 2005. The one large cell neuroendocrine carcinoma was resected at Narita Red Cross Hospital in 2004. The tumors in all cases were resected completely. We performed immunohistochemical staining for all large cell neuroendocrine carcinomas, small cell carcinomas, classic large cell carcinomas, and carcinoid tumors studied. Neuroendocrine differentiation was detected for large cell neuroendocrine carcinoma by positive immunohistochemical staining for chromogranin A, synaptophysin, or CD56. We confirmed lack of neuroendocrine differentiation in all classic large cell carcinomas. Two experienced pulmonary pathologists ( $\mathrm{KH}$ and $\mathrm{YN}$ ) classified the tumors independently. Discordant independent readings were resolved by simultaneous review performed by the two readers. Informed consent was obtained from all patients.

One of 12 patients with classic large cell carcinoma, and seven of 23 with small cell carcinoma underwent preoperative chemotherapy. One patient with classic large cell carcinoma, five with large cell neuroendocrine carcinoma, and 13 with small cell carcinoma underwent postoperative adjuvant chemotherapy, and one patient with large cell neuroendocrine carcinoma and two with small cell carcinoma underwent postoperative radiotherapy.

\section{Morphometric Study}

The nuclear diameter was measured in each case of large cell neuroendocrine carcinoma and small cell carcinoma with a CAS 200 cellular imaging system using a $\times 40$ objective lens (Becton Dickinson and Company, San Jose, CA, USA). The accuracy of the image measurement was confirmed with the standard objective micrometer (Nikon, Tokyo, Japan). The nuclear diameters of 100 tumor cells and the nuclear diameters of 20 mature lymphocytes were measured for each specimen. The measurements were made by one of the authors $(\mathrm{KH})$. The mean and standard deviation were then calculated.

\section{Immunohistochemistry}

All tumors were examined by immunohistochemical staining. Sections $(4 \mu \mathrm{m})$ were cut from 
formalin-fixed paraffin-embedded tissues and placed on silanized slides (DakoCytomation, Glostrup, Denmark). They were stained with polyclonal anti-chromogranin A antibody (prediluted; Nichirei, Tokyo, Japan), polyclonal anti-synaptophysin antibody (prediluted; DakoCytomation), polyclonal anti-Neuro $D$ antibody (1:200; N-19, Santa Cruz Biotechnology, Santa Cruz, CA, USA), polyclonal anti-phosphatase and tensin homologue (PTEN) antibody (1:200; PN37, Zymed Laboratories, South San Francisco, CA, USA), monoclonal anti-CD56 antibody (prediluted; 123C3, Zymed Laboratories), monoclonal anti-human cytokeratin high molecular weight (CK34 $\beta$ E12) antibody (prediluted; 34 $\beta$ E12, DakoCytomation), monoclonal anti-thyroid transcription factor-1 (TTF-1) antibody (1:200; 8G7G3/1, DakoCytomation), monoclonal anti-p63 protein antibody $(1: 400 ; 4 \mathrm{~A} 4$, DakoCytomation), and monoclonal anti-p16 protein antibody (1:50; G175-405, Pharmingen, San Diego, CA, USA). We used monoclonal anti-mASH1 antibody (1:50; 24B72D11.1, Becton Dickinson Biosciences, Franklin Lakes, NJ, USA) to detect $h A S H 1$ because this antibody crossreacts with hASH1 of human small cell carcinoma in the paraffin-embedded tissues. ${ }^{12,13}$

We used a Histofine Simple Stain Kit (Nichirei, Tokyo, Japan) for immunostaining. To improve the staining pattern, the tissues were pretreated with microwaves for $15 \mathrm{~min}$ in citrate buffer $(10 \mathrm{mM}$ $\mathrm{pH}$ 6.0) before staining with anti-CD56 antibody, anti-CK34 $\beta$ E12 antibody, anti-Neuro $D$ antibody, and anti-p16 antibody; or were heated in an autoclave at $121^{\circ} \mathrm{C}$ for $15 \mathrm{~min}$ before staining with anti-synaptophysin antibody, anti-PTEN antibody, anti-TTF-1 antibody, and anti-mASH1 antibody. The tissues were heated in an autoclave at $121^{\circ} \mathrm{C}$ for $15 \mathrm{~min}$ in DAKO Target Retrieval Solution (S1700) (DakoCytomation) before staining with anti-p63 protein antibody. Reactivity was considered negative if less than $10 \%$ of tumor cells stained.

\section{Microdissection and DNA Extraction}

DNA was extracted from paraffin-embedded materials as described previously. ${ }^{10}$

\section{Polymorphic DNA Markers and Polymerase Chain Reaction-Loss of Heterozygosity}

We examined previously the same cohort for the loss of heterozygosity (LOH) at 13 microsatellite markers: D3S1234(3p14.2), D3S1481(3p14.2), D3S1295(3p21.1), D3S1581(3p21.3), D5S407(5q11), D5S410(5q31.3), D5S422(5q33), D9S171(9p21), IFNA(9p21), D10S249 (10p15.3), D10S1686(10q22.3), D13S153(13q14), and ALE3/P53ivs1b(TP53). LOH was defined as a reduction of an allele's peak height by at least $50 \%$ in the tumor, compared with the normal sample. ${ }^{10}$

\section{Calculation of Fractional Regional Loss Index}

The fractional regional loss index was calculated as follows:

Fractional regional loss $=$ (total number of chromosomal regions with $\mathrm{LOH}) /($ total number of informative regions).

\section{Methylation Analysis}

We examined previously the same cohort for the methylation state of the $p 16$ gene by methylationspecific polymerase chain reaction (PCR). ${ }^{10,14}$

\section{Survival Analysis}

Because all cases with classic large cell carcinoma, large cell neuroendocrine carcinoma, and carcinoid tumors and seven of 23 cases with small cell carcinoma were in stage I, survival of these cases was determined.

The interval between the date of surgery and the date of the first local and distant recurrence was defined as disease-free survival, and the time after surgery until death was defined as the overall survival. Kaplan-Meier curves and survival estimates were calculated, ${ }^{15}$ and the Brestow-GehanWilcoxon test was used to test for differences between groups.

\section{Statistical Analysis}

All statistical calculations were made with the StatView software package (SAS Institute Inc., Cary, NC, USA). Differences of the ratio of the nuclear diameter of the tumor cells to that of the lymphocytes between large cell neuroendocrine carcinoma and small cell carcinoma were analyzed by Student's $t$-test. A $\chi^{2}$ test was used to evaluate the immunohistochemical and methylation analysis for the lung cancer subtypes and the differences of the frequency of $\mathrm{LOH}$ at specific regions among lung cancer subtypes. Differences in fractional regional loss indices among three lung cancer subtypes were tested by the Kruskal-Wallis test, and those between large cell neuroendocrine carcinoma and small cell carcinoma were tested by Mann-Whitney $U$-test. Probability values of $P<0.05$ were regarded as statistically significant.

\section{Results}

\section{Microscopic Study}

Examples of histological preparations of small cell carcinoma and large cell neuroendocrine carcinoma are presented in Figure 1a and b. The tumor cells of large cell neuroendocrine carcinoma are polygonal with a low nuclear to cytoplasmic (N/C) ratio; are larger than small cell carcinoma cells; have 

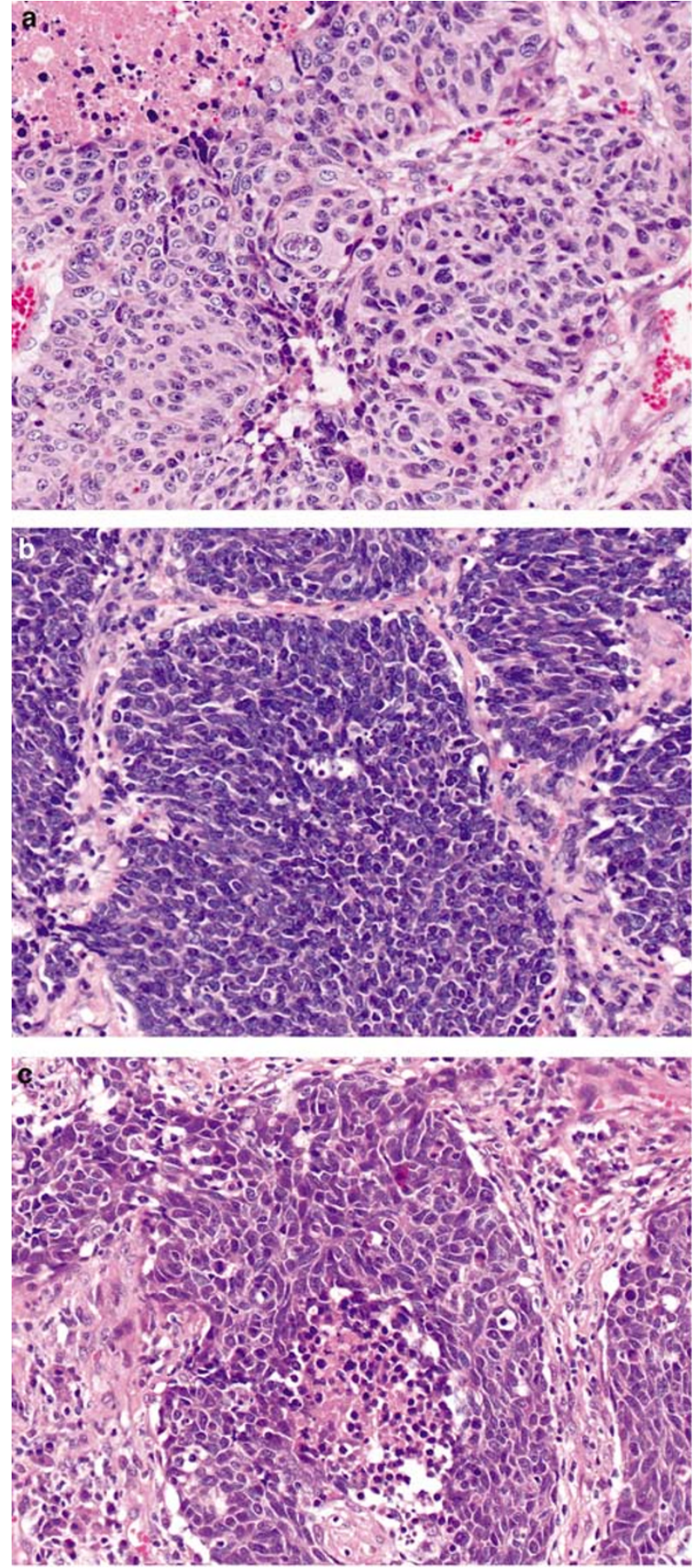

Figure 1 (a) Large cell neuroendocrine carcinoma. This tumor has organoid nesting and a rosette arrangement. Tumor cells have abundant eosinophilic cytoplasm, coarsely granular chromatin, and prominent nucleoli. Tumor cells with larger nuclei are observed. Necrosis is present. (b) Small cell carcinoma. Cell size is small. Tumor cells have scant cytoplasm so that the cellularity is high. The nuclei are round or oval. The chromatin of the nucleus is vesicular, and nucleoli are inconspicuous. (c) Small cell carcinoma. Cell size is larger than in (b), but smaller than in (a). Tumor cells are polygonal and have a moderate amount of cytoplasm. Rosette-like structures are present. The chromatin of the nucleus is vesicular, and nucleoli are seen. Necrosis is present. moderate to abundant eosinophilic cytoplasm; have a high mitotic rate (11 or more mitotic figures per 10 high power fields), prominent nucleoli, and coarsely granular nuclear chromatin. A large infarct-like zone of necrosis is present in large cell neuroendocrine carcinoma (Figure 1a). Small cell carcinoma is typically composed of small (less than the size of three small resting lymphocytes), round to fusiform cells with a high N/C ratio. The cells have a high mitotic rate, absent or inconspicuous nucleoli, and finely granular nuclear chromatin (Figure 1b). Some cases of small cell carcinoma have somewhat different cytological features. The cell size appears larger than that of typical small cell carcinoma (Figure 1c). Tumor cells have polygonal shape and a small amount of cytoplasm. The nuclei are relatively large, and the N/C ratio was high. The nuclear chromatin is coarsely granular or vesicular. The nucleoli are usually observed but are small or inconspicuous. The mitosis number is high. The cell border is sharp in one area but unclear in another.

In two cases with combined small cell carcinoma and squamous cell carcinoma, the predominant histological type was squamous cell carcinoma, in one case, the predominant histological type was small cell carcinoma. In a case with combined small cell carcinoma and large cell neuroendocrine carcinoma, the predominant histological type was large cell neuroendocrine carcinoma, and in a case with combined large cell neuroendocrine carcinoma and squamous cell carcinoma, the predominant histological type was large cell neuroendocrine carcinoma.

\section{Morphometric Study}

The frequency distribution of tumor nuclear diameter/lymphocyte size ratios is shown in a histogram (Figure 2a and b). The peak of tumor nuclear diameter/lymphocyte size ratios was between 3 and 4 for large cell neuroendocrine carcinoma and between 2 and 3 for small cell carcinoma. The average tumor nuclear diameter/lymphocyte size ratios were $3.22 \pm 0.86$ for large cell neuroendocrine carcinoma and $2.75 \pm 0.86$ for small cell carcinoma. The differences between tumor nuclear diameter/ lymphocyte size ratios of large cell neuroendocrine carcinoma and small cell carcinoma were significant $(P<0.0001)$ according to the Student's $t$-test.

\section{Immunohistochemistry}

Immunohistochemical study showed that 59\% of large cell neuroendocrine carcinoma and $36 \%$ of small cell carcinoma stained with antibody for chromogranin A $(P=0.1630)$. Seventy seven percent of large cell neuroendocrine carcinoma and $57 \%$ of small cell carcinoma stained with antibody for synaptophysin $(P=0.1910)$. Fifty three percent of large cell neuroendocrine carcinoma and $96 \%$ of small cell carcinoma stained with antibody for CD56 

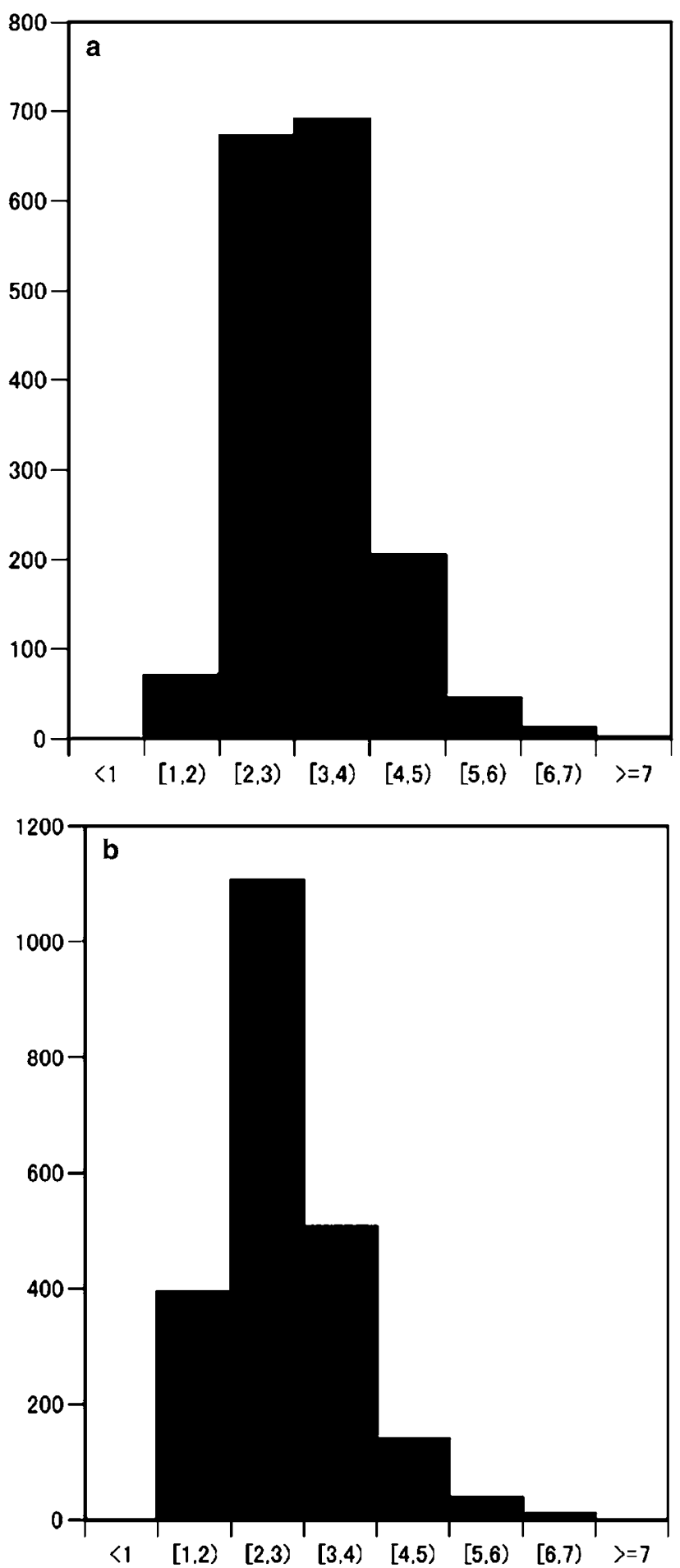

Figure 2 The frequency distribution of tumor nuclear diameter/ lymphocyte size ratios. The nuclear diameters of 100 tumor cells and the nuclear diameters of 20 mature lymphocytes were measured for each specimen. The tumor nuclear diameter/ lymphocyte size ratio of each tumor cell was calculated as the nuclear diameter of each tumor cell divided by the mean nuclear diameter of lymphocytes. A histogram was made of all tumor nuclear diameter/lymphocyte size ratios measured in each subtype. (a) Large cell neuroendocrine carcinoma. The peak of the tumor nuclear diameter/lymphocyte size ratio is between 3 and 4. (b) Small cell carcinoma. The peak of the tumor nuclear diameter/lymphocyte size ratio is between 2 and 3 .

Table 1 Summary of immunohistochemical staining for neuroendocrine markers

\begin{tabular}{lrrr}
\hline & \multicolumn{1}{c}{ LCNEC } & \multicolumn{1}{c}{ SCLC } & \\
\hline Chromogranin & $10 / 17(59 \%)$ & $8 / 22(36 \%)$ & 0.1630 \\
Synaptophysin & $13 / 17(77 \%)$ & $13 / 23(57 \%)$ & 0.1910 \\
CD56 & $9 / 17(53 \%)$ & $21 / 22(96 \%)$ & 0.0018
\end{tabular}

LCNEC, large cell neuroendocrine carcinoma; SCLC, small cell lung carcinoma.

$(P=0.0018)$ (Table 1$)$. Fifteen of 17 pure large cell neuroendocrine carcinomas and 19 of 23 small cell carcinomas were completely CK34 $\beta$ E12 negative. However, tumor cells of two of pure large cell neuroendocrine carcinomas and four of small cell carcinomas were stained focally with antiCK34 $\beta$ E12. In large cell neuroendocrine carcinomas and small cell carcinomas combined with squamous cell carcinoma, the last component showed marked immunoreactivity with CK34 $\beta$ E12.

The cytoplasm of bronchial gland cells was stained positively for $m A S H 1$ in normal lung tissue, and no cells of the bronchial epithelium were stained; both cytoplasm and nucleus of the tumor cells were stained. A few cells in normal bronchial epithelium had cytoplasmic granules that stained positively for NeuroD; both cytoplasm and nucleus of tumor cells were stained with that antibody. Type II alveolar epithelial cells in normal lung tissue were stained with the antibody for TTF-1; only nuclei of tumor cells were stained. Nuclei of basal cells of bronchial epithelium were stained with anti-p63 antibody, and only nuclei of tumor cells were stained. Tumor cells were stained focally with anti-p63 antibody in four small cell carcinomas, and one carcinoid tumor. Because we defined negative reactivity as fewer than $10 \%$ of tumor cells stained, these cases were negative for $p 63$. This pattern contrasted with tumor cells that were stained uniformly in classic large cell carcinoma and large cell neuroendocrine carcinoma. Only cells with nuclear activity for anti-p16 antibody were recorded. Prostatic hyperplasia tissue was used for the positive control for PTEN; both cytoplasm and nucleus of the tumor cell stained with the antibody.

Table 2 lists the total number and percentage of positive cases in each histological category for each antibody. For cases with combined small cell carcinoma with non-small-cell carcinoma, immunohistochemical staining of the small cell carcinoma is described in Table 2, and for a case with combined large cell neuroendocrine carcinoma, that for the large cell neuroendocrine carcinoma is described. The differences of the frequency of the expressions of mASH1, NeuroD, TTF-1, p63, p16, and PTEN were statistically significant among classic large cell carcinoma, large cell neuroendocrine carcinoma, small cell carcinoma, and carcinoid tumors. The differences of staining between high-grade neuroendocrine carcinoma were analyzed. The 
Table 2 Summary of immunohistochemical staining results

\begin{tabular}{|c|c|c|c|c|c|c|}
\hline & $C L C C$ & & SCLC & Carcinoid & \multicolumn{2}{|c|}{$\mathrm{P}$-value } \\
\hline mASH1 & $0 / 12(0 \%)$ & 10/17 (59\%) & $20 / 23(87 \%)$ & $0 / 9(0 \%)$ & $<0.0001^{\mathrm{a}}$ & $0.0422^{b}$ \\
\hline NeuroD & $4 / 12(33 \%)$ & 10/17 (59\%) & $3 / 23(13 \%)$ & $5 / 9(56 \%)$ & 0.0139 & 0.0022 \\
\hline TTF-1 & $4 / 12(33 \%)$ & $4 / 17(24 \%)$ & $13 / 23(57 \%)$ & $0 / 9(0 \%)$ & 0.0141 & 0.0369 \\
\hline p63 & $6 / 12(50 \%)$ & $3 / 17(18 \%)$ & $1 / 23(4 \%)$ & $0 / 9(0 \%)$ & 0.0028 & 0.1657 \\
\hline p16 & $7 / 12(58 \%)$ & 10/17 (59\%) & $21 / 23(91 \%)$ & $2 / 9(22 \%)$ & 0.0019 & 0.0150 \\
\hline PTEN & $9 / 12(75 \%)$ & $5 / 17(29 \%)$ & $2 / 23(9 \%)$ & $9 / 9(100 \%)$ & $<0.0001$ & 0.0883 \\
\hline
\end{tabular}

CLCC, classic large cell carcinoma; LCNEC, large cell neuroendocrine carcinoma; SCLC, small cell lung carcinoma.

${ }^{\mathrm{a}}$ Difference of the frequency of expression among four subtypes of lung carcinoma.

${ }^{\mathrm{b}}$ Difference of the frequency of expression among high-grade neuroendocrine carcinoma of the lung.

Table 3 Results of immunohistochemical results in combined SCLC and combined LCNEC

\begin{tabular}{|c|c|c|c|c|c|c|}
\hline Case & $m A S H 1$ & NeuroD & $T T F-1$ & p63 & p16 & PTEN \\
\hline \multirow{2}{*}{\multicolumn{7}{|c|}{ Combined SCLC }} \\
\hline & & & & & & \\
\hline SCLC & + & - & + & - & + & - \\
\hline SQ & - & + & - & + & + & + \\
\hline \multicolumn{7}{|l|}{2} \\
\hline SCLC & + & - & + & - & + & - \\
\hline SQ & - & - & - & + & - & \pm \\
\hline \multicolumn{7}{|l|}{3} \\
\hline SCLC & + & + & + & - & - & - \\
\hline SQ & - & - & - & + & - & \pm \\
\hline \multicolumn{7}{|l|}{4} \\
\hline SCLC & + & - & + & + & + & - \\
\hline LCNEC & - & - & + & - & + & \pm \\
\hline \multicolumn{7}{|c|}{$\begin{array}{l}\text { Combined LCNEC } \\
1\end{array}$} \\
\hline LCNEC & - & + & - & - & + & \pm \\
\hline SQ & - & + & - & + & - & + \\
\hline
\end{tabular}

SCLC, component of small cell lung carcinoma; LCNEC, component of large cell neuroendocrine carcinoma; SQ, component of sqamous cell carcinoma; +, positive; - , negative; \pm , decreased.

frequencies of the expressions of mASH1, TTF-1, and $p 16$ were higher in small cell carcinoma than in large cell neuroendocrine carcinoma, and those of the expressions of NeuroD, p63, and PTEN were higher in large cell neuroendocrine carcinoma than in small cell carcinoma. The differences of the frequency of the expressions of $m A S H 1$, NeuroD, TTF-1, and $p 16$ were statistically significant between high-grade neuroendocrine carcinoma. The results of immunohistochemical staining for combined neuroendocrine carcinomas according to each component are shown in Table 3. mASH1 was expressed only in the component of small cell carcinoma in all cases of combined small cell carcinoma and squamous cell carcinoma. TTF-1 was expressed only in the component of small cell carcinoma in all cases of combined small cell carcinoma and squamous cell carcinoma and in both components in one case of combined small cell carcinoma and large cell neuroendocrine carcinoma.

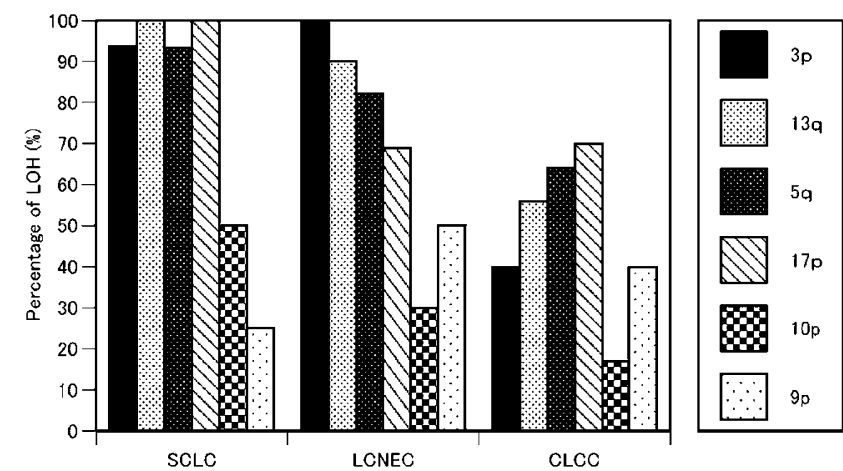

Figure 3 Relations between percentage of $\mathrm{LOH}$ by chromosomal regions and histologic categories. SCLC, small cell lung carcinoma; LCNEC, large cell neuroendocrine carcinoma; CLCC, classic large cell carcinoma.

\section{Polymorphic DNA Markers and PCR-LOH}

Four specific 3p regions (D3S1234, D3S1481, D3S1295, and D3S1581), three specific 5q regions (D5S407, D5S410, and D5S422), two specific 9p regions (D9S171 and IFNA), one specific 10p region (D10S249), one specific 10q region (D10S1686), one specific $13 q$ region (D13S153), and one specific $17 p$ region (ALE3/P53ivs1b) were analyzed. The frequencies of $\mathrm{LOH}$ in specific regions are shown in Figure 3. The frequency of $\mathrm{LOH}$ at $10 \mathrm{q}$ is not shown because the number of informative cases at $10 \mathrm{q}$ was small. The frequency of $\mathrm{LOH}$ at $3 p$ was higher in high-grade neuroendocrine carcinoma than in classic large cell carcinoma and the difference was statistically significant $(P=0.0002)$. The frequency of $\mathrm{LOH}$ at $13 \mathrm{q}$ was also higher in high-grade neuroendocrine carcinoma than in classic large cell carcinoma, but the difference was not statistically significant. The frequency of $\mathrm{LOH}$ at $5 \mathrm{q}, 17 \mathrm{p}$, and $10 \mathrm{p}$ was higher in small cell carcinoma than in large cell neuroendocrine carcinoma and classic large cell carcinoma, and the frequency of $\mathrm{LOH}$ at $9 p$ was higher in large cell neuroendocrine carcinoma and classic large cell carcinoma than in small cell carcinoma, but the differences were not significant. Allelic losses at D5S422 were observed in nine of 11 cases of small cell carcinoma (82\%) and in one of six cases of large cell neuroendocrine carcinoma (17\%). 
The difference in frequency between small cell carcinoma and large cell neuroendocrine carcinoma was statistically significant $(P=0.0091)$. Allelic losses at ALE3/P53ivs1b were observed in of all of seven cases of small cell carcinoma $(100 \%)$ and in eight of 12 cases of large cell neuroendocrine carcinoma $(67 \%)$. However, the difference in frequency between small cell carcinoma and large cell neuroendocrine carcinoma was not statistically significant $(P=0.0856)$.

Mean fractional regional loss indices of the tumors were $0.38,0.65$, and 0.72 for patients with classic large cell carcinoma, large cell neuroendocrine carcinoma, and small cell carcinoma, respectively. The differences of fractional regional loss indices among the three types of the tumor were significant $(P=0.0003)$; however, those between high-grade neuroendocrine carcinomas were not $(P=0.1123)$. Mean $3 p$ fractional regional loss indices of the tumor were $0.20,0.76$, and 0.79 for patients with classic large cell carcinoma, large cell neuroendocrine carcinoma, and small cell carcinoma, respectively. The differences of fractional regional loss indices among the three types of tumor were significant $(P=0.0004)$; however, those between high-grade neuroendocrine carcinomas were not $(P=0.5689)$.

\section{Methylation Analysis}

Hypermethylation of the $p 16$ gene was observed in four of 12 informative cases of classic large cell carcinoma (33\%), three of 10 informative cases of large cell neuroendocrine carcinoma (30\%), and three of 14 informative cases of small cell carcinoma $(21 \%)$. The difference of the frequency was not significant $(P=0.7825)$.

\section{Survival Analysis}

Five-year overall survivals of patients with classic large cell carcinoma, large cell neuroendocrine carcinoma, small cell carcinoma, and carcinoid tumors were $64,73,60$, and $89 \%$, respectively. The differences of overall survival among the five subtypes were not significant. Five-year disease-free survivals of patients with classic large cell carcinoma, large cell neuroendocrine carcinoma, small cell carcinoma, and carcinoid tumors were 64, 58, 62, and $89 \%$, respectively. The differences of disease-free survival among the five subtypes were not significant.

We examined the correlations between survival of patients with high-grade neuroendocrine carcinoma and the expressions of the proteins examined in this study. Comparing patients with/without a specific protein expression, patients with NeuroD expression had better 5-year disease-free $(80 / 46 \%$, $P=0.0336)$ and overall $(91 / 50 \%, P=0.0355)$ survivals. Patients with $m A S H 1$ expression had poorer 5-year disease-free $(46 / 80 \%, P=0.2016)$ and overall $(60 / 80 \%, P=0.4395)$ survivals, but the difference was not significant. Patients with p63 expression had poorer 5-year disease-free $(33 / 61 \%, P=0.0583)$ and overall $(33 / 71 \%, P=0.0428)$ survivals. Patients with TTF-1 expression had poorer 5-year diseasefree $(23 / 69 \%, P=0.0419)$ and overall $(19 / 81 \%$, $P=0.0117$ ) survivals. Other biomarkers did not have prognostic significance for either disease-free or overall survivals in high-grade neuroendocrine carcinoma.

We examined subset analysis of the correlations between survival of patients with high-grade neuroendocrine carcinoma and the expressions of the proteins. Patients with NeuroD expression had better 5-year disease-free $(74 / 33 \%, P=0.0413)$ and overall $(89 / 50 \%, P=0.0887)$ survivals in large cell neuroendocrine carcinoma. Patients with mASH1 expression had poorer 5-year overall survival (48/ $100 \%$ ) in small cell carcinoma, but the difference was not significant. Patients with p63 expression had poorer 5-year disease-free $(33 / 64 \%$, $P=0.0394)$ and overall $(33 / 83 \%, P=0.0157)$ survivals (Figure 4a) in large cell neuroendocrine carcinoma. Patients with TTF-1 expression had poorer 5-year disease-free $(0 / 77 \%, P=0.0269)$ and overall $(0 / 77 \%, P=0.0208)$ survivals in small cell carcinoma (Figure 4b).
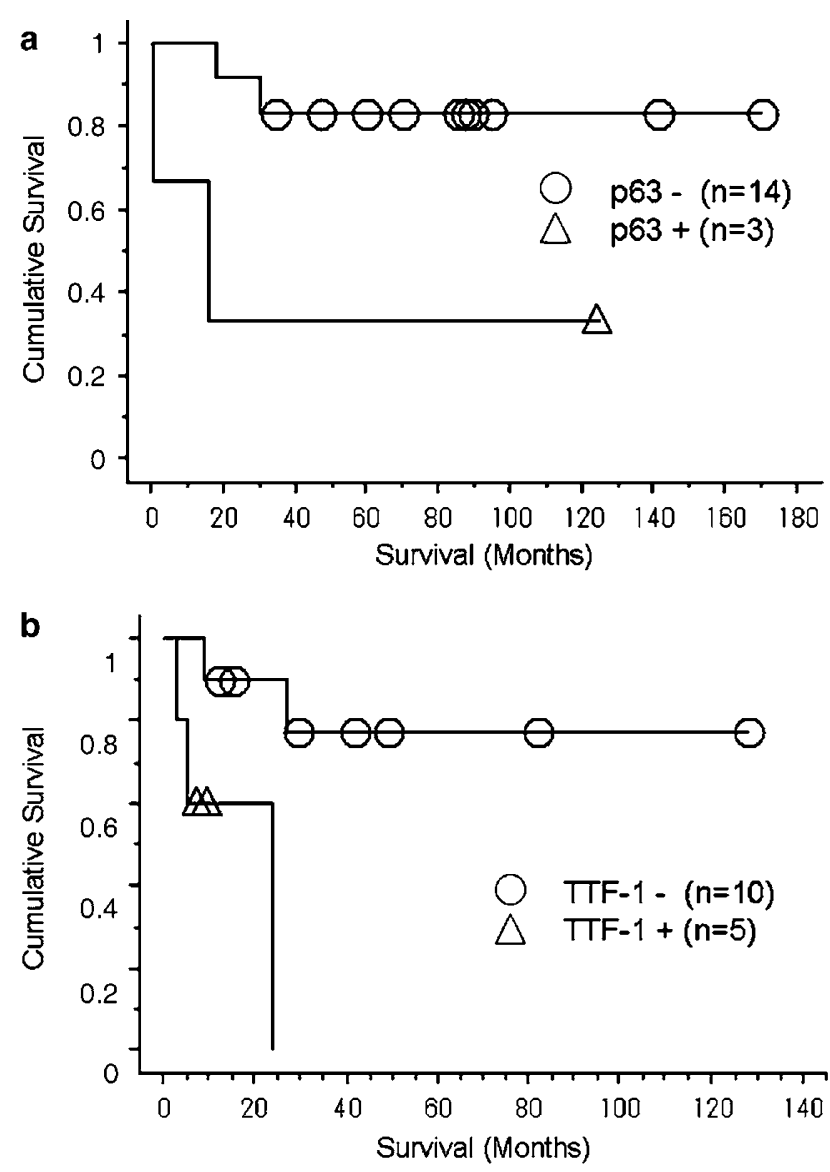

Figure 4 (a) The overall survival curves of p63-positive and p63-negative large cell neuroendocrine carcinoma cases. (b) The overall survival curves of cases with TTF-1-positive and TTF1-negative small cell carcinoma. 


\section{Discussion}

Achaete-scute homolog 1 (termed mASH1 in rodents, hASH1 in humans) is a basic helix-loophelix (bHLH) transcription factor that is important in the early development of neural and neuroendocrine progenitor cells in many tissues. ${ }^{16}$ The NeuroD gene is a bHLH gene and regulates neurogenesis, and it is reported that it maps to chromosome 2q32. ${ }^{17}$ NeuroD is expressed transiently in developing neurons in the central and peripheral nervous systems but is not expressed in nearby neuronal progenitors that are still undergoing cell division. ${ }^{18}$ $m A S H 1$ is required at an early stage in neuronal differentiation and initiates a differentiation program involving NeuroD. ${ }^{19}$

It is reported that $h A S H 1$ is expressed selectively in normal fetal pulmonary neuroendocrine cells ${ }^{20}$ as well as in pulmonary large cell neuroendocrine carcinoma $(56.7 \%)$ and small cell carcinoma $(71.8 \%){ }^{21}$ The frequency of expression of $m A S H 1$ in our study was $59 \%$ in large cell neuroendocrine carcinoma and $87 \%$ in small cell carcinoma. The frequencies of expression of $\mathrm{mASH} 1$ in large cell neuroendocrine carcinoma and small cell carcinoma are comparable to those reported in a previous paper. ${ }^{21}$ It was reported that the expression of NeuroD was detected in $24.3 \%$ of gastric adenocarcinomas, but not in gastric neuroendocrine carcinomas, including small cell carcinomas. ${ }^{22}$ The expression of NeuroD in our study was $59 \%$ in large cell neuroendocrine carcinoma and $13 \%$ in small cell carcinoma. The frequency of the expression of NeuroD was higher in large cell neuroendocrine carcinoma, but it was also observed in carcinoid tumor and classic large cell carcinoma. NeuroD may be related to neuroendocrine differentiation in nonsmall-cell carcinoma including large cell neuroendocrine carcinoma. It was reported that $h A S H 1$ expression was associated with a poorer prognosis in small cell carcinoma patients. ${ }^{21}$ In our study, the relation between the survival of patients and the expression of $m A S H 1$ was not significant; however, NeuroD expression was associated with good prognosis in large cell neuroendocrine carcinoma.

A putative tumor suppressor gene, PTEN, was identified at $10 \mathrm{q} 23$ and is thought to play an important role in the control of cell proliferation, apoptosis, differentiation, ${ }^{23,24}$ and break cell cycle progression by inhibiting the phosphatidylinositol $3^{\prime}$-kinase pathway. ${ }^{25,26}$ PTEN was mutated or deleted homozygously in glioma, endometrial, and breast carcinoma tumors. ${ }^{27-29}$ It was reported that PTEN mutations were identified in small cell carcinomas, but not in non-small-cell carcinomas. ${ }^{30,31}$ We found that the frequency of the loss of expression of PTEN was higher in high-grade neuroendocrine carcinoma than in classic large cell carcinoma or carcinoid tumor, and the differences were significant; however, the difference was not significant between large cell neuroendocrine carcinoma and small cell carcinoma.
TTF-1 is a tissue-specific transcription factor that mediates cell determination and differentiation in thyroid, lung, and brain. It is expressed in thyroid follicular cells, human fetal lung, and alveolar type II epithelial cells after birth. TTF-1 was detectable in pulmonary adenocarcinomas, ${ }^{32,33}$ small cell carcinomas, large cell neuroendocrine carcinomas, carcinoid tumors, and classic large cell carcinomas but not in pulmonary squamous cell carcinomas. ${ }^{33,34}$ The prognostic value of TTF-1 was evaluated in non-small-cell carcinomas, and contradictory results were reported. ${ }^{35,36}$ We found that TTF-1 was detectable in $33 \%$ of classic large cell carcinoma cases, $24 \%$ of large cell neuroendocrine carcinoma, and $57 \%$ in small cell carcinoma. TTF-1 had a poor prognostic implication in small cell carcinoma in our study.

p63 is a p53 homologue mapped to chromosome 3q27-28. p63 plays a role in stem cell commitment in squamous epithelium. ${ }^{37}$ Some reported that $p 63$ was expressed in pulmonary squamous cell carcinoma but not in small cell carcinomas, ${ }^{37,38}$ whereas others reported that large cell neuroendocrine carcinoma and small cell carcinoma had p63 expression. ${ }^{39,40}$ The reported differences of frequency of p63 expression in small cell carcinoma may be due to different identification methods for positive cells. Zhang et $a l^{38}$ reported that they defined the tumor as p63-negative if less than $10 \%$ of tumor cells were stained. Pelosi et $a l^{39}$ reported that they defined the tumor as p63-positive if nuclear staining of the tumor cells was observed. It is reported that p63 staining cases have a better outcome in patients with squamous cell carcinoma ${ }^{41,42}$ but a poor outcome in patients with neuroendocrine tumors. ${ }^{40}$ In our study, half of classic large cell carcinoma and $18 \%$ of large cell neuroendocrine carcinoma cases stained with antip63 antibody, but $4 \%$ of small cell carcinoma cases was p63-positive. Patients with p63 expression had poorer 5-year overall survival $(33 \%)$ than those without its expression (83\%) in large cell neuroendocrine carcinoma $(P=0.0157)$.

Recently, a genome-wide high-resolution search for LOH was made of small cell carcinoma and nonsmall-cell carcinoma cell lines. ${ }^{43,44}$ These studies found that some LOH was common in both small cell carcinoma and non-small-cell carcinoma subtypes, whereas loss was subtype-specific in others. It has been suggested that the genetic alterations undergone by small cell carcinoma and non-smallcell carcinoma are different. There are contradictory reports about the differences of genetic changes between small cell carcinoma and large cell neuroendocrine carcinoma. For example, $\mathrm{LOH}$ at $3 p$, $5 q, 11 q, 13 q, 17 p$ and mutations in the p53 and ras genes were present both in small cell carcinomas and large cell neuroendocrine carcinomas. ${ }^{45}$ $c$-myc amplification was observed with a similar frequency in small cell carcinoma $(20 \%)$ and large cell neuroendocrine carcinoma $(23 \%) .{ }^{46}$ Small cell 
carcinoma and large cell neuroendocrine carcinoma were indistinguishable by gene expression profiles, and high-grade neuroendocrine carcinoma of the lung can be classified into two groups independent of small cell carcinoma and large cell neuroendocrine carcinoma. ${ }^{8}$ However, although both small cell carcinoma and large cell neuroendocrine carcinoma shared several chromosomal aberrations including losses of $3 p, 4 q, 5 q$, and $13 q$ and gains of $5 p$, a gain of $3 q$ and a loss of 10q, 16q, and 17p were observed frequently in small cell carcinoma but not in large cell neuroendocrine carcinoma, and a gain of $6 \mathrm{p}$ occurred more frequently in large cell neuroendocrine carcinoma. ${ }^{47}$ Our study found that the frequency of $\mathrm{LOH}$ at $3 p$ and $13 q$ was high in large cell neuroendocrine carcinoma and small cell carcinoma, it was higher at $5 q$ and $17 p$ in small cell carcinoma than in large cell neuroendocrine carcinoma, and it was higher at $9 p$ in large cell neuroendocrine carcinoma than in small cell carcinoma. Allelic losses at D5S422 were more frequent in small cell carcinoma than in large cell neuroendocrine carcinoma $(P=0.0091)$. We conclude that there are different genetic changes in small cell carcinoma and large cell neuroendocrine carcinoma and also common abnormalities between these two subtypes.

Chromogranin A and synaptophysin are the most reliable immunohistochemical markers to detect neuroendocrine differentiation in neuroendocrine lung tumors. ${ }^{3,4,48}$ CD56 or neural cell adhesion molecule is also a sensitive and specific marker in confirmation of neuroendocrine differentiation in malignant neoplasms. ${ }^{48,49}$ It is reported that CD56 is useful for the immunohistochemical differentiation of small cell carcinoma and large cell neuroendocrine carcinoma from nonneuroendocrine carcionomas. ${ }^{49-51}$ The frequencies of the expressions of CD56 were higher in small cell carcinoma (96\%) than in large cell neuroendocrine carcinoma (53\%), and the difference was statistically significant in our study.

Large cell neuroendocrine carcinoma is defined as a large cell carcinoma showing histological features such as organoid nesting, trabecular, rosette-like and palisading patterns; and neuroendocrine differentiation confirmed by immunohistochemistry or electron microscopy. ${ }^{3,11}$ The tumor cells are polygonal with a low N/C ratio; are larger than small cell carcinoma cells; have moderate to abundant eosinophilic cytoplasm; have a high mitotic rate (11 or more mitotic figures per 10 high-power fields), prominent nucleoli, and coarsely granular nuclear chromatin. A large infarct-like zone of necrosis is present in large cell neuroendocrine carcinoma. Small cell carcinoma is composed of small (less than the size of three small resting lymphocytes), round to fusiform cells with a high N/C ratio. ${ }^{3,11}$ The cells have a high mitotic rate, absent or inconspicuous nucleoli, and finely granular nuclear chromatin.
It is reported that there exist borderline cases between large cell neuroendocrine carcinoma and small cell carcinoma in high-grade neuroendocrine carcinoma. ${ }^{52}$ Difficulty of diagnosis in high-grade neuroendocrine carcinoma is thought to occur as a result of variety of reasons, including the continuum of cell size and morphology between small cell carcinoma and large cell neuroendocrine carcinoma, and poor sampling and tissue artifacts. ${ }^{53}$ We propose that one of the main reasons why there are differences between observers in histological diagnosis of high-grade neuroendocrine carcinoma may be the presence of tumors shown in Figure 1c in this study. We diagnose this tumor as small cell carcinoma because the N/C ratio is high and the cytoplasm is not abundant. However, some pathologists may diagnose the same specimen as large cell neuroendocrine carcinoma because the shape of the tumor cell is polygonal, the nuclear diameter is larger than that of typical small cell carcinoma, and nucleoli are usually observed. The average tumor nuclear diameter/lymphocyte size ratios in tumors shown in Figure 1c is 2.91, and significantly larger than that in typical small cell carcinoma (2.62). Tumors as shown in Figure 1c have a unique immunohistochemical phenotype, such that all tumors are CD56 positive, chromogranin A negative, and synaptophysin negative $(n=10)$. Methylation of $p 16$ gene is observed in half of the tumors shown in Figure 1c, but not observed in typical small cell carcinoma. However, the smaller nuclear size, the expression of CD56, mASH1, and p16, and the $\mathrm{LOH}$ analysis have supported the recognition that tumors as shown in Figure 1c are small cell carcinoma.

In the present study, the 5-year overall survivals of patients with classic large cell carcinoma, large cell neuroendocrine carcinoma, small cell carcinoma, and carcinoid tumors were $64,73,60$, and $89 \%$, respectively. The differences of overall survival among the four subtypes were not significant. Fiveyear survival for small cell carcinoma in this study was good, because we evaluated the survival of small cell carcinoma in stage I. These cases were operated in early stage and underwent preoperative and/or postoperative chemotherapy. We had previously analyzed small cell carcinoma resected surgically at our institute and reported that the 5 -year overall survival for patients with small cell carcinoma in all stages was $16.4 \% .^{54}$

In summary, we found that antibodies to CD56, mASH1, NeuroD, TTF-1, and p16 made up a useful panel of immunohistochemical markers for the differential diagnosis of large cell neuroendocrine carcinoma and small cell carcinoma, and that NeuroD expression correlated with a good survival and p63 with a poor survival in patients with large cell neuroendocrine carcinoma, and TTF-1 with a poor survival in patients with small cell carcinoma. Additional studies in larger series are needed to analyze the biological behavior of 
large cell neuroendocrine carcinoma and small cell carcinoma including sensitivity to chemotherapeutic agents.

\section{Acknowledgements}

We thank Ms Tamiyo Taniguchi, Ms Ayaka Sato, and Ms Kazuko Abe for their technical assistance. This study was partially supported by the Smoking Research Foundation.

\section{References}

1 World Health Organization. Histological Typing of Lung Tumours. World Health Organization: Geneva, 1967.

2 World Health Organization. Histological Typing of Lung Tumours, 2nd edn. World Health Organization: Geneva, 1981.

3 World Health Organization. Histological Typing of Lung and Pleural Tumours, 3rd edn. Springer-Verlag: Berlin, 1999.

4 Travis WD, Linnoila RI, Tsokos MG, et al. Neuroendocrine tumors of the lung with proposed criteria for large-cell neuroendocrine carcinoma. An ultrastructural, immunohistochemical, and flow cytometric study of 35 cases. Am J Surg Pathol 1991;15:529-553.

5 Travis WD, Gal AA, Colby TV, et al. Reproducibility of neuroendocrine lung tumor classification. Hum Pathol 1998;29:272-279.

6 Marchevsky AM, Gal AA, Shah S, et al. Morphometry confirms the presence of considerable nuclear size overlap between 'small cells' and 'large cells' in highgrade pulmonary neuroendocrine neoplasms. Am J Clin Pathol 2001;116:466-472.

7 Wick MR. Neuroendocrine neoplasia. Current concepts. Am J Clin Pathol 2000;113:331-335.

8 Jones $\mathrm{MH}$, Virtanen $\mathrm{C}$, Honjoh $\mathrm{D}$, et al. Two prognostically significant subtypes of high-grade lung neuroendocrine tumours independent of small-cell and large-cell neuroendocrine carcinomas identified by gene expression profiles. Lancet 2004;363:775-781.

9 Iyoda A, Hiroshima K, Toyozaki T, et al. Adjuvant chemotherapy for large cell carcinoma with neuroendocrine features. Cancer 2001;92:1108-1112.

10 Hiroshima K, Iyoda A, Shibuya K, et al. Genetic alterations in early-stage pulmonary large cell neuroendocrine carcinoma. Cancer 2004;100:1190-1198.

11 Travis WD, Brambilla E, Muller-Hermelink HK, et al. World Health Organization Classification of Tumour. Pathology and Genetics of Tumours of the Lung, Pleura, Thymus and Heart. IARC Press: Lyon, 2004.

$12 \mathrm{Hu}$ Y, Ippolito JE, Garabedian EM, et al. Molecular characterization of a metastatic neuroendocrine cell cancer arising in the prostates of transgenic mice. J Biol Chem 2002;277:44462-44474.

13 Shida T, Furuya M, Nikaido T, et al. Aberrant expression of human achaete-scute homologue gene 1 in the gastrointestinal neuroendocrine carcinomas. Clin Cancer Res 2005;11:450-458.

14 Herman JG, Graff JR, Myohanen S, et al. Methylationspecific PCR: a novel PCR assay for methylation status of CpG islands. Proc Natl Acad Sci USA 1996;93: 9821-9826.
15 Kaplan EL, Meier P. Nonparametric estimation from incomplete observations. J Am Stat Assoc 1958;53: 457-481.

16 Ball DW. Achaete-scute homolog-1 and Notch in lung neuroendocrine development and cancer. Cancer Lett 2004;204:159-169.

17 Tamimi R, Steingrimsson E, Copeland NG, et al. The NEUROD gene maps to human chromosome 2q32 and mouse chromosome 2. Genomics 1996;34:418-421.

18 Lee JE, Hollenberg SM, Snider L, et al. Conversion of Xenopus ectoderm into neurons by NeuroD, a basic helix-loop-helix protein. Science 1995;268:836-844.

19 Cau E, Gradwohl G, Fode C, et al. Mash1 activates a cascade of bHLH regulators in olfactory neuron progenitors. Development 1997;124:1611-1621.

20 Borges M, Linnoila RI, van de Velde HJ, et al. An achaete-scute homologue essential for neuroendocrine differentiation in the lung. Nature 1997;386:852-855.

21 Jiang SX, Kameya $\mathrm{T}$, Asamura $\mathrm{H}$, et al. hASH1 expression is closely correlated with endocrine phenotype and differentiation extent in pulmonary neuroendocrine tumors. Mod Pathol 2004;17:222-229.

22 Fujii A, Kamiakito T, Takayashiki N, et al. Neuroendocrine tissue-specific transcription factor, BETA2/ NeuroD, in gastric carcinomas: a comparison with chromogranin A and synaptophysin expressions. Pathol Res Pract 2003;199:513-519.

$23 \mathrm{Lu} \mathrm{Y,} \mathrm{Lin} \mathrm{YZ,} \mathrm{LaPushin} \mathrm{R,} \mathrm{et} \mathrm{al.} \mathrm{The} \mathrm{PTEN/MMAC1/}$ TEP tumor suppressor gene decreases cell growth and induces apoptosis and anoikis in breast cancer cells. Oncogene 1999;18:7034-7045.

24 Tian XX, Pang JC, To SS, et al. Restoration of wild-type $P T E N$ expression leads to apoptosis, induces differentiation, and reduces telomerase activity in human glioma cells. J Neuropathol Exp Neurol 1999;58: 472-479.

25 Myers MP, Pass I, Batty IH, et al. The lipid phosphatase activity of PTEN is critical for its tumor suppressor function. Proc Natl Acad Sci USA 1998;95:1351313518.

26 Maehama T, Dixon JE. PTEN: a tumour suppressor that functions as a phospholipid phosphatase. Trends Cell Biol 1999;9:125-128.

27 Chen J, Lindblom P, Lindblom A. A study of the PTEN/ $M M A C 1$ gene in 136 breast cancer families. Hum Genet 1998;102:124-125.

28 Davies MP, Gibbs FE, Halliwell N, et al. Mutation in the PTEN/MMAC1 gene in archival low grade and high grade gliomas. Br J Cancer 1999;79:1542-1548.

29 Bussaglia E, del Rio E, Matias-Guiu X, et al. PTEN mutations in endometrial carcinomas: a molecular and clinicopathologic analysis of 38 cases. Hum Pathol 2000;31:312-317.

30 Yokomizo A, Tindall DJ, Drabkin $\mathrm{H}$, et al. PTEN/ MMAC1 mutations identified in small cell, but not in non-small cell lung cancers. Oncogene 1998;17: 475-479.

31 Wang L, Ignat A, Axiotis CA. Differential expression of the PTEN tumor suppressor protein in fetal and adult neuroendocrine tissues and tumors: progressive loss of PTEN expression in poorly differentiated neuroendocrine neoplasms. Appl Immunohistochem Mol Morphol 2002;10:139-146.

32 Bejarano PA, Baughman RP, Biddinger PW. Surfactant proteins and thyroid transcription factor-1 in pulmonary and breast carcinomas. Mod Pathol 1996;9: 445-452. 
33 Kaufmann O, Dietel M. Expression of thyroid transcription factor-1 in pulmonary and extrapulmonary small cell carcinomas and other neuroendocrine carcinomas of various primary sites. Histopathology 2000;36:415-420.

34 Folpe AL, Gown AM, Lamps LW, et al. Thyroid transcription factor-1: immunohistochemical evaluation in pulmonary neuroendocrine tumors. Mod Pathol 1999;12:5-8.

35 Puglisi F, Barbone F, Damante G, et al. Prognostic value of thyroid transcription factor-1 in primary, resected, non-small cell lung carcinoma. Mod Pathol 1999;12: 318-324.

36 Myong NH. Thyroid transcription factor-1 (TTF-1) expression in human lung carcinomas: its prognostic implication and relationship with expressions of p53 and Ki-67 proteins. J Korean Med Sci 2003;18:494-500.

37 Wang BY, Gil J, Kaufman D, et al. P63 in pulmonary epithelium, pulmonary squamous neoplasms, and other pulmonary tumors. Hum Pathol 2002;33:921-926.

38 Zhang $\mathrm{H}$, Liu J, Cagle PT, et al. Distinction of pulmonary small cell carcinoma from poorly differentiated squamous cell carcinoma: an immunohistochemical approach. Mod Pathol 2005;18:111-118.

39 Pelosi G, Pasini F, Olsen Stenholm C, et al. p63 immunoreactivity in lung cancer: yet another player in the development of squamous cell carcinomas? J Pathol 2002;198:100-109.

$40 \mathrm{Au} \mathrm{NH}$, Gown AM, Cheang M, et al. P63 expression in lung carcinoma: a tissue microarray study of 408 cases. Appl Immunohistochem Mol Morphol 2004;12: 240-247.

41 Massion PP, Taflan PM, Jamshedur Rahman SM, et al. Significance of p63 amplification and overexpression in lung cancer development and prognosis. Cancer Res 2003;63:7113-7121.

$42 \mathrm{Au} \mathrm{NH}$, Cheang M, Huntsman DG, et al. Evaluation of immunohistochemical markers in non-small cell lung cancer by unsupervised hierarchical clustering analysis: a tissue microarray study of 284 cases and 18 markers. J Pathol 2004;204:101-109.

43 Virmani AK, Fong KM, Kodagoda D, et al. Allelotyping demonstrates common and distinct patterns of chromosomal loss in human lung cancer types. Genes Chromosomes Cancer 1998;21:308-319.
44 Girard L, Zochbauer-Muller S, Virmani AK, et al. Genome-wide allelotyping of lung cancer identifies new regions of allelic loss, differences between small cell lung cancer and non-small cell lung cancer, and loci clustering. Cancer Res 2000;60:4894-4906.

45 Onuki N, Wistuba II, Travis WD, et al. Genetic changes in the spectrum of neuroendocrine lung tumors. Cancer 1999;85:600-607.

46 Gugger M, Burckhardt E, Kappeler A, et al. Quantitative expansion of structural genomic alterations in the spectrum of neuroendocrine lung carcinomas. J Pathol 2002;196:408-415.

47 Ullmann R, Petzmann S, Sharma A, et al. Chromosomal aberrations in a series of large-cell neuroendocrine carcinomas: unexpected divergence from small-cell carcinoma of the lung. Hum Pathol 2001;32:10591063.

48 Brambilla E, Veale D, Moro D, et al. Neuroendocrine phenotype in lung cancers. Comparison of immunohistochemistry with biochemical determination of enolase isoenzymes. Am J Clin Pathol 1992; 98:88-97.

49 Kaufmann O, Georgi T, Dietel M. Utility of 123C3 monoclonal antibody against CD56 (NCAM) for the diagnosis of small cell carcinomas on paraffin sections. Hum Pathol 1997;28:1373-1378.

50 Kontogianni K, Nicholson AG, Butcher D, et al. CD56: a useful tool for the diagnosis of small cell lung carcinomas on biopsies with extensive crush artefact. J Clin Pathol 2005;58:978-980.

51 Lantuejoul S, Moro D, Michalides RJ, et al. Neural cell adhesion molecules (NCAM) and NCAM-PSA expression in neuroendocrine lung tumors. Am J Surg Pathol 1998;22:1267-1276.

52 Asamura H, Kameya T, Matsuno Y, et al. Neuroendocrine neoplasms of the lung: a prognostic spectrum. J Clin Oncol 2006;24:70-76.

53 Nicholson SA, Beasley MB, Brambilla E, et al. Small cell lung carcinoma (SCLC): a clinicopathologic study of 100 cases with surgical specimens. Am J Surg Pathol 2002;26:1184-1197.

54 Iyoda A, Hiroshima K, Baba M, et al. Pulmonary large cell carcinomas with neuroendocrine features are high-grade neuroendocrine tumors. Ann Thorac Surg 2002;73:1049-1054. 